August 2002

Volume 110 | Number 4

\title{
The role of intestinal P-glycoprotein in the interaction of digoxin and rifampin.
}

\author{
Michel Eichelbaum, Bernd Greiner, Peter Fritz, Hans-Peter Kreichgauer, \\ Oliver von Richter, Johannes Zundler, Heyo K. Kroemer
}

J. Clin. Invest. 104:147-153 (1999).

In our $1999 \mathrm{JCI}$ article, we reported that the rate and extent of absorption of orally administered digoxin is determined by the level of intestinal P-glycoprotein (P-gp) expression. Rifampin treatment reduced digoxin plasma concentrations substantially after oral administration and, to a lesser extent, after intravenous (i.v.) administration. Moreover, rifampin increased intestinal P-gp content $3.5 \pm 2.1$-fold which correlated significantly with the area under the plasma concentration curve under pharmacokinetic calculations up to 144 hours $\left(\mathrm{AUC}_{0-144 \mathrm{~h}}\right)$ after oral digoxin.

Based on these findings, we concluded that intestinal P-gp affects the extent of digoxin biovailability and that the decreasing oral digoxin bioavailability during rifampin is caused by induction of intestinal P-gp.

Two issues were brought to our attention by Win $\mathrm{L}$. Chiou, Sang M. Chung and Ta C. Wu (College of Pharmacy

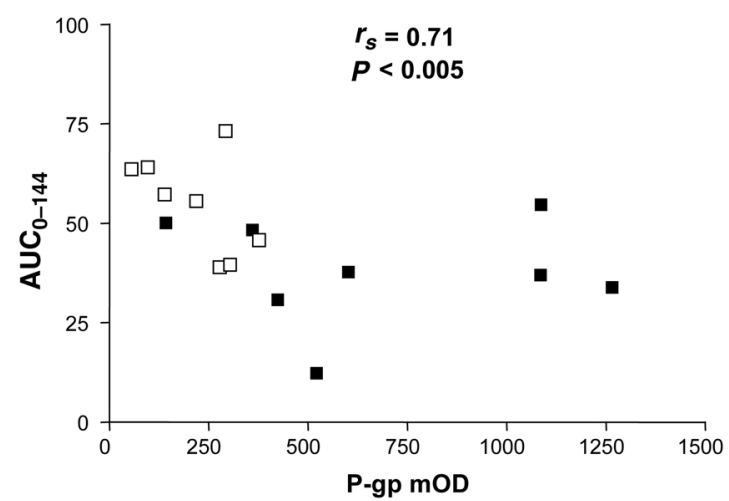

Figure 2

(c) Correlation between AUC of orally administered digoxin (1 mg) and expression of P-gP $(n=16)$ measured by Western blot. Open squares, without rifampin; filled squares, with rifampin (600 mg).

Table 3

Expression of CYP3A and P-gp in enterocytes of duodenal biopsies before $(n=8)$ and after $(n=8)$ administration of rifampin, as determined by Western blot

\begin{tabular}{|c|c|c|c|c|}
\hline & $\begin{array}{c}\text { Control } \\
{[\mathrm{OD}]}\end{array}$ & $\begin{array}{c}\text { With rifampin } \\
\text { [OD] }\end{array}$ & $\begin{array}{c}\text { Ratio } \\
\text { Rifampin/control }\end{array}$ & $P$ value \\
\hline CYP3A & $91 \pm 40$ & $369 \pm 250$ & $4.4 \pm 2.7$ & $P<0.005$ \\
\hline P-gp & $220 \pm 113$ & $685 \pm 407$ & $3.5 \pm 2.1$ & $P<0.005$ \\
\hline
\end{tabular}

at the University of Illinois at Chicago). Chiou et al. had requested and received the original study data from us. The input of Chiou and colleagues is appreciated.

During compilation of the data for Greiner et al., data pairs comparing AUC digoxin versus P-gp/CYP3A levels from the control and rifampin period were transposed and misassigned. For this reason, as Chiou et al. recognized, Figure $2 \mathrm{c}$ and Table 3 of our publication required revision. The corrected data are shown below.

Our recalculation with the correct assigned data pairs confirms the initial observation of a significant relationship between $\mathrm{AUC}_{0-144 \mathrm{~h}}$ and P-gp levels and result in relatively minor changes in Spearman rank correlation from $r_{s} 0.78$. to $r_{s} 0.71$ and significance with $P<0.005$ instead of $P<0.0005$ (Figure 2c). The mean values of P-gP and CYP3A in the revised Table 3 are somewhat different from the initial reported data, but this has no effect on the ratios of rifampin/control or the significance level.

A second concern raised by Chiou et al. relates to the calculation of oral digoxin bioavailability AUC. It was not explicitly stated in the article that for the calculation of oral digoxin bioavailability $\mathrm{AUC}_{0-144 \mathrm{~h}}$ and not $\mathrm{AUC}_{0-00}$ was used and $\mathrm{AUC}_{\mathrm{i} . \mathrm{v}}$ from the control period was used to derive oral digoxin bioavailability during rifampin.

Our original calculation of oral bioavailability was based on the $\mathrm{AUC}_{0-144 \mathrm{~h}}$ because, beyond this time point, digoxin plasma levels were below the limit of quantification under conditions of rifampin treatment. Since rifampin also affects the i.v. disposition of digoxin, probably in part by direct intestinal secretion by $\mathrm{P}-\mathrm{gp}$, the $\mathrm{AUC}_{\mathrm{iv}}$ from the control period was used. However, calculating bioavailability with $\mathrm{AUC}_{\mathrm{i} . \mathrm{.}}$ from rifampin, oral digoxin $\mathrm{F}$ is $49.5 \%$ as compared to $44 \%$ with $\mathrm{AUC}_{\text {i.v. }}$ from the control period. Although there are some differences between the initially published and recalculated values, the principal findings and conclusions of our study are not invalidated. 